\title{
A Weyl-Titchmarsh type formula for a discrete Schrödinger operator with Wigner-von Neumann potential
}

by

\author{
Jan Janas (Kraków) and Sergey Simonov (St. Petersburg)
}

\begin{abstract}
We consider a discrete Schrödinger operator $\mathcal{J}$ with Wigner-von Neumann potential not belonging to $l^{2}$. We find the asymptotics of orthonormal polynomials associated to $\mathcal{J}$. We prove a Weyl-Titchmarsh type formula, which relates the spectral density of $\mathcal{J}$ to a coefficient in the asymptotics of the orthonormal polynomials.
\end{abstract}

1. Introduction. In recent papers on Jacobi matrices [6], [13, [12, [24], 23], 22 new results were found on the asymptotics of generalized eigenvectors of these operators. For real sequences $\left\{a_{n}\right\}_{n=1}^{\infty}$ and $\left\{b_{n}\right\}_{n=1}^{\infty}$ the Jacobi operator $J=J\left(a_{n}, b_{n}\right)$ is defined in the Hilbert space $l^{2}$ by the formula

$$
(J u)_{n}=a_{n-1} u_{n-1}+b_{n} u_{n}+a_{n} u_{n+1} .
$$

It is well known that the spectral analysis of Jacobi operators is closely related to the study of the asymptotics of generalized eigenvectors. In this work we concentrate on the discrete Schrödinger operator $\mathcal{J}=\mathcal{J}\left(1, b_{n}\right)$ with the Wigner-von Neumann potential

$$
\begin{gathered}
b_{n}=\frac{c \sin (2 \omega n+\delta)}{n^{\gamma}}+q_{n}, \\
\gamma \in(1 / 3 ; 1 / 2), \quad 2 \omega \notin \pi \mathbb{Z}, \quad\left\{q_{n}\right\}_{n=1}^{\infty} \in l^{1},
\end{gathered}
$$

where $c, \omega, \delta, q_{n} \in \mathbb{R}$. The Jacobi operator $\mathcal{J}$ is given by

$$
\begin{aligned}
& (\mathcal{J} u)_{1}=b_{1} u_{1}+u_{2}, \\
& (\mathcal{J} u)_{n}=u_{n-1}+b_{n} u_{n}+u_{n+1}, \quad n \geq 2,
\end{aligned}
$$

2010 Mathematics Subject Classification: Primary 47B36; Secondary 34E10.

Key words and phrases: Jacobi matrices, asymptotics of generalized eigenvectors, orthogonal polynomials, Weyl-Titchmarsh theory, discrete Schrödinger operator, Wigner-von Neumann potential. 
and has a matrix representation

$$
\mathcal{J}=\left(\begin{array}{cccc}
b_{1} & 1 & 0 & \cdots \\
1 & b_{2} & 1 & \cdots \\
0 & 1 & b_{3} & \ldots \\
\vdots & \vdots & \vdots & \ddots
\end{array}\right)
$$

in the canonical basis $\left\{e_{n}\right\}_{n=1}^{\infty}$ of $l^{2}$. Since $\mathcal{J}$ is a compact perturbation of the free discrete Schrödinger operator its essential spectrum is the interval $[-2 ; 2]$. The frequency $2 \omega$ in the potential produces (in general) four critical (or resonance) points inside this interval: $\pm 2 \cos \omega, \pm 2 \cos 2 \omega$. At these points the resonance occurs and the asymptotics of the generalized eigenvectors changes (the analogous phenomenon in the continuous case is very well studied: see [9], [7, Chapter 4] and [19, Theorem 5]) and an eigenvalue can appear under certain additional conditions.

In the present paper we are interested in the asymptotics of the orthogonal polynomials $P_{n}(\lambda)$ associated to $\mathcal{J}$, which are

$$
\begin{aligned}
P_{1}(\lambda) & :=1, \quad P_{2}(\lambda):=\lambda-b_{1}, \\
P_{n+1}(\lambda) & :=\left(\lambda-b_{n}\right) P_{n}(\lambda)-P_{n-1}(\lambda), \quad n \geq 2,
\end{aligned}
$$

and the relation of this asymptotics to the spectral density $\rho^{\prime}(\lambda)$ of $\mathcal{J}$.

Our main result (see Theorem 5.1 on page 180 and Theorem 5.3 on page 185 for the exact formulation) is that there exists a function $F$ (the Jost function) such that for a.a. $\lambda \in(-2 ; 2)$,

$$
\rho^{\prime}(\lambda)=\frac{\sqrt{4-\lambda^{2}}}{2 \pi|F(z)|^{2}}
$$

and

$$
\begin{aligned}
P_{n}(\lambda)= & \frac{z F(z)}{1-z^{2}} \frac{1}{z^{n}} \exp \left(\frac{\mu_{2}(z) n^{1-2 \gamma}}{1-2 \gamma}\right) \\
& +\frac{z \overline{F(z)}}{z^{2}-1} z^{n} \exp \left(-\frac{\mu_{2}(z) n^{1-2 \gamma}}{1-2 \gamma}\right)+o(1) \quad \text { as } n \rightarrow \infty,
\end{aligned}
$$

where

$$
\lambda=z+\frac{1}{z}, \quad z=\frac{\lambda-i \sqrt{4-\lambda^{2}}}{2}
$$

and

$$
\mu_{2}(z):=\frac{c^{2} z^{2}\left(1+z^{2}\right) e^{2 i \omega}}{4\left(1-z^{2}\right)\left(z^{2}-e^{2 i \omega}\right)\left(1-z^{2} e^{2 i \omega}\right)} .
$$

This result is an analog of the classical Weyl-Titchmarsh (or Kodaira) formula for the differential Schrödinger operator on the half-line with summable potential (see [27, Chapter 5] and [16]). 
We consider $\gamma \in(1 / 3 ; 1 / 2)$ for the following reasons. If $\gamma>1 / 2$, then $\left\{b_{n}\right\}_{n=1}^{\infty} \in l^{2}$ and the sum $\sum_{n=1}^{\infty} b_{n}$ converges. This situation was studied using a completely different method by Damanik and Simon in [6] for a class of more general Jacobi matrices, with an arbitrary sequence $\left\{b_{n}\right\}_{n=1}^{\infty} \in l^{2}$ for which $\sum_{n=1}^{\infty} b_{n}$ is convergent. In this case the asymptotics of the polynomials $P_{n}(\lambda)$ is simpler (of Szegö type):

$$
P_{n}(\lambda)=\frac{z F(z)}{1-z^{2}} \frac{1}{z^{n}}+\frac{z \overline{F(z)}}{z^{2}-1} z^{n}+o(1) \quad \text { as } n \rightarrow \infty,
$$

and this was proven in [6, Theorem 8.1] not for a.a. $\lambda \in(-2 ; 2)$, but in the sense of convergence in $L_{2}\left((-2 ; 2), \rho^{\prime}(x) d x\right)$. If one proves that 1.6 holds for a.a. $\lambda \in(-2 ; 2)$ (which for the potential of the form (1.1) is much simpler than the analysis that we develop in the present paper), then (1.4) can be deduced from results of Damanik and Simon ([6, Theorems 5.6 and 8.1]) in a non-trivial way (this was pointed out by Dr. Roman Romanov in a private communication). On the other hand, the type of the asymptotics for $\gamma \in$ $(1 / 2 ; 1]$ is the same as in the simple case $\gamma>1$. For $\gamma=1 / 2$ the type of the asymptotics changes. This happens also for every $\gamma=1 / l, l \in \mathbb{N} \backslash\{1\}$. We are forced to consider a different (depending on $l$ ) number of terms in the asymptotic expansion that we use (see (3.7)). The greater $l$ is, the more terms are significant. Our method works for every $\gamma>0$, but we restrict ourselves to the case $\gamma \in(1 / 3 ; 1 / 2)$. However, in the final section we state the corresponding result for $\gamma \in(1 / 2 ; 1]$ (Theorem 6.1) and indicate how to simplify the proof for that case. The main idea of the method is inspired by [5] and uses the discrete version of a change of variables introduced by Eastham in [7].

We could as well consider a finite sum of terms like (1.1) as the potential,

$$
b_{n}=\sum_{l=1}^{L} \frac{c_{l} \sin \left(2 \omega_{l} n+\delta_{l}\right)}{n^{\gamma_{l}}}+q_{n},
$$

with the same conditions imposed on $c_{l}, \omega_{l}, \gamma_{l}, \delta_{l}$ and $\left\{q_{n}\right\}_{n=1}^{\infty}$. This would increase the number of critical points and complicate the notation and calculations, so we restrict ourselves to the case of one such term only.

After this work was sent to publication we learned about the work of Lukic [20] who studied a 1D Schrödinger operator $H$ with a more general potential given by a sequence in $l^{p}(p \in \mathbb{N})$ which is of generalized bounded variation. In particular he proved that the spectral measure of $H$ has no singular continuous part and its point spectrum in $(-2 ; 2)$ is contained in a specific finite set (which in our case is exactly the set of critical points).

In the continuous case (a differential Schrödinger operator on the halfline) Wigner-von Neumann potentials were studied in numerous works: [21], [2], 3], [10], 15], 17], 18], [5], 19], and formulas for the spectral density analogous to $(1.4)$ were obtained in different variations in [21], [2] and [5]. 
The Weyl-Titchmarsh type formula can be used to study the spectral density of the Jacobi matrix $\mathcal{J}$. The type of the asymptotics of orthogonal polynomials changes at critical points, and analyzing the process of this change it is possible to prove that the spectral density has zeros at critical points. For the case $\gamma=1$, in the forthcoming paper we plan to prove the following. Let $\nu_{\text {cr }}$ be one of the critical points $\pm 2 \cos \omega$. If $\left\{P_{n}\left(\nu_{\mathrm{cr}}\right)\right\}_{n=1}^{\infty}$ is not a subordinate solution of the spectral equation, then both one-sided limits

$$
\lim _{\lambda \rightarrow \nu_{\mathrm{cr}} \pm 0} \frac{\rho^{\prime}(\lambda)}{\left|\lambda-\nu_{\mathrm{cr}}\right|^{\frac{|c|}{2|\sin \omega|}}}
$$

exist. In the case $\gamma<1$ the zeros of the spectral density at critical points presumably have exponential type, but this problem seems to be much more difficult than in the case $\gamma=1$.

2. Preliminaries. For every complex $\lambda$ the spectral equation for $\mathcal{J}$,

$$
u_{n-1}+b_{n} u_{n}+u_{n+1}=\lambda u_{n}, \quad n \geq 2,
$$

has solutions $P_{n}(\lambda)$ (orthogonal polynomials of the first kind) and $Q_{n}(\lambda)$ (orthogonal polynomials of the second kind) such that

$$
\begin{aligned}
P_{1}(\lambda) & =1, & P_{2}(\lambda) & =\lambda-b_{1}, \\
Q_{1}(\lambda) & =0, & Q_{2}(\lambda) & =1 .
\end{aligned}
$$

For non-real $\lambda$ there exists $m(\lambda)$ (the Weyl function) such that

$$
Q_{n}(\lambda)+m(\lambda) P_{n}(\lambda)
$$

is the unique (up to multiplication by a constant) solution of (2.1) that belongs to $l^{2}$ (see [1]). The Weyl function and the spectral density of $\mathcal{J}$ are related by the following equalities:

$$
\begin{aligned}
& m(\lambda)=\int_{\mathbb{R}} \frac{d \rho(x)}{x-\lambda}, \quad \lambda \in \mathbb{C} \backslash \mathbb{R}, \\
& \rho^{\prime}(\lambda)=\frac{1}{\pi} \operatorname{Re} m(\lambda+i 0) \quad \text { for a.a. } \lambda \in \mathbb{R} .
\end{aligned}
$$

For any two solutions $u$ and $v$ of 2.1 with the same parameter $\lambda$ the (discrete) Wronskian defined by

$$
W(u, v):=u_{n} v_{n+1}-u_{n+1} v_{n}
$$

is independent of $n$. For the polynomials $P(\lambda)$ and $Q(\lambda)$ the Wronskian is equal to one for every $\lambda$.

3. Reduction of the spectral equation to a system of L-diagonal form. Consider the spectral equation for $\mathcal{J}$,

$$
u_{n-1}+b_{n} u_{n}+u_{n+1}=\lambda u_{n}, \quad n \geq 2 .
$$


Let us write it in vector form:

$$
\left(\begin{array}{c}
u_{n} \\
u_{n+1}
\end{array}\right)=\left(\begin{array}{cc}
0 & 1 \\
-1 & \lambda-b_{n}
\end{array}\right)\left(\begin{array}{c}
u_{n-1} \\
u_{n}
\end{array}\right), \quad n \geq 2 .
$$

Consider a new parameter $z \in \overline{\mathbb{D}}$ (we denote by $\mathbb{D}$ the open unit disc) such that

and conversely

$$
\lambda=z+\frac{1}{z}
$$

$$
z=\frac{\lambda-i \sqrt{4-\lambda^{2}}}{2}
$$

where the branch of the square root is chosen so that $z \in \mathbb{D}$ for $\lambda \in \mathbb{C} \backslash[-2 ; 2]$, i.e., $z=-i$ for $\lambda=0$. Let

$$
v_{n}:=\frac{z}{z^{2}-1}\left(\begin{array}{cc}
z & -1 \\
-1 / z & 1
\end{array}\right)\left(\begin{array}{c}
u_{n} \\
u_{n+1}
\end{array}\right),
$$

which is equivalent to

$$
\left(\begin{array}{c}
u_{n} \\
u_{n+1}
\end{array}\right)=\left(\begin{array}{cc}
1 & 1 \\
1 / z & z
\end{array}\right) v_{n}, \quad n \geq 1 .
$$

We make this substitution to diagonalize the constant part of the coefficient matrix:

$$
\left(\begin{array}{cc}
0 & 1 \\
-1 & \lambda
\end{array}\right)=\left(\begin{array}{cc}
1 & 1 \\
1 / z & z
\end{array}\right)\left(\begin{array}{cc}
1 / z & 0 \\
0 & z
\end{array}\right)\left(\begin{array}{cc}
1 & 1 \\
1 / z & z
\end{array}\right)^{-1}
$$

Equation $(3.2)$ becomes

$$
v_{n+1}=\left[\left(\begin{array}{cc}
1 / z & 0 \\
0 & z
\end{array}\right)+\frac{b_{n+1}}{z^{2}-1}\left(\begin{array}{cc}
1 & z^{2} \\
-1 & -z^{2}
\end{array}\right)\right] v_{n}, \quad n \geq 1 .
$$

The goal of the present section is to reduce the system (3.3) to an "Ldiagonal" form. If we put

$$
w_{n}:=T_{n}^{-1}(z) v_{n}
$$

then $(3.3)$ becomes

$$
w_{n+1}=T_{n+1}^{-1}(z)\left[\left(\begin{array}{cc}
1 / z & 0 \\
0 & z
\end{array}\right)+\frac{b_{n+1}}{z^{2}-1}\left(\begin{array}{cc}
1 & z^{2} \\
-1 & -z^{2}
\end{array}\right)\right] T_{n}(z) w_{n} .
$$

The system is in L-diagonal form if the coefficient matrix is a sum of diagonal and summable matrices. So we have to find matrices $T_{n}(z)$ to provide this property. This is possible not for every $z \neq 0,1,-1$.

Let us denote

$$
U:=\mathbb{C} \backslash\left\{0,1,-1, e^{ \pm i \omega},-e^{ \pm i \omega}, e^{ \pm 2 i \omega},-e^{ \pm 2 i \omega}\right\} .
$$


Lemma 3.1. Let $z \in U$. For every $n \in \mathbb{N}$ there exist matrices $R_{n}^{(2)}(z)$ and invertible matrices $T_{n}(z)$ such that

$$
\begin{aligned}
& T_{n}(z), T_{n}^{-1}(z)=I+o(1), \\
& R_{n}^{(2)}(z)=O\left(\frac{1}{n^{3 \gamma}}+\left|q_{n+1}\right|\right)
\end{aligned}
$$

as $n \rightarrow \infty$ and

$$
\begin{aligned}
T_{n+1}^{-1}(z)\left[\left(\begin{array}{cc}
1 / z & 0 \\
0 & z
\end{array}\right)+\right. & \left.\frac{b_{n+1}}{z^{2}-1}\left(\begin{array}{cc}
1 & z^{2} \\
-1 & -z^{2}
\end{array}\right)\right] T_{n}(z) \\
& =\left(\begin{array}{cc}
\frac{1}{z}\left(1+\frac{\mu_{2}(z)}{n^{2 \gamma}}\right) & 0 \\
0 & z\left(1-\frac{\mu_{2}(z)}{n^{2 \gamma}}\right)
\end{array}\right)+R_{n}^{(2)}(z),
\end{aligned}
$$

where $\mu_{2}(z)$ is given by 1.5$) \cdot T_{n}(z)$ and $R_{n}^{(2)}(z)$ are also analytic in $U$ for all $n$, and on every compact subset of $U$ the estimate (3.5) is uniform with respect to $z$.

Proof. Let us denote

$$
\begin{aligned}
\Lambda(z) & :=\left(\begin{array}{cc}
1 / z & 0 \\
0 & z
\end{array}\right), & N_{2}(z) & :=\frac{c e^{i(2 \omega+\delta)}}{2 i\left(z^{2}-1\right)}\left(\begin{array}{cc}
1 & z^{2} \\
-1 & -z^{2}
\end{array}\right), \\
R_{n}^{(0)}(z) & :=\frac{q_{n+1}}{z^{2}-1}\left(\begin{array}{cc}
1 & z^{2} \\
-1 & -z^{2}
\end{array}\right), & N_{-2}(z) & :=-\frac{c e^{-i(2 \omega+\delta)}}{2 i\left(z^{2}-1\right)}\left(\begin{array}{cc}
1 & z^{2} \\
-1 & -z^{2}
\end{array}\right),
\end{aligned}
$$

so that

$$
\frac{b_{n+1}}{z^{2}-1}\left(\begin{array}{cc}
1 & z^{2} \\
-1 & -z^{2}
\end{array}\right)=\frac{e^{2 i \omega n}}{n^{\gamma}} N_{2}(z)+\frac{e^{-2 i \omega n}}{n^{\gamma}} N_{-2}(z)+R_{n}^{(0)}(z) .
$$

We will find $T_{n}(z)$ in two steps.

The first (and main) step of the construction is to find matrices $T_{n}^{(1)}(z)$ such that

$$
\begin{array}{r}
\left(T_{n+1}^{(1)}(z)\right)^{-1}\left[\left(\begin{array}{cc}
1 / z & 0 \\
0 & z
\end{array}\right)+\frac{b_{n+1}}{z^{2}-1}\left(\begin{array}{cc}
1 & z^{2} \\
-1 & -z^{2}
\end{array}\right)\right] T_{n}^{(1)}(z) \\
=\left(\begin{array}{cc}
1 / z & 0 \\
0 & z
\end{array}\right)+\frac{V(z)}{n^{2 \gamma}}+R_{n}^{(1)}(z)
\end{array}
$$

where $V(z)$ does not depend on $n$ and $R_{n}^{(1)}(z)$ is summable. 
Following the ideas of [7] and [5] we look for $T_{n}^{(1)}(z)$ of the form

$$
\begin{aligned}
T_{n}^{(1)}(z):=\exp \left(\frac{e^{2 i \omega n}}{n^{\gamma}} X_{2}(z)+\right. & \frac{e^{-2 i \omega n}}{n^{\gamma}} X_{-2}(z) \\
& \left.+\frac{e^{4 i \omega n}}{n^{2 \gamma}} X_{4}(z)+\frac{e^{-4 i \omega n}}{n^{2 \gamma}} X_{-4}(z)\right),
\end{aligned}
$$

where $X_{ \pm 2}(z)$ and $X_{ \pm 4}(z)$ are to be determined. Define

$$
\begin{aligned}
M_{ \pm 4}:= & \frac{1}{2}\left(\Lambda X_{ \pm 2}^{2}+e^{ \pm 4 i \omega} X_{ \pm 2}^{2} \Lambda\right)+N_{ \pm 2} X_{ \pm 2} \\
& -e^{ \pm 2 i \omega} X_{ \pm 2} N_{ \pm 2}-e^{ \pm 2 i \omega} X_{ \pm 2} \Lambda X_{ \pm 2}
\end{aligned}
$$

and

(3.9) $\quad V:=\frac{1}{2}\left(\Lambda\left(X_{2} X_{-2}+X_{-2} X_{2}\right)+\left(X_{2} X_{-2}+X_{-2} X_{2}\right) \Lambda\right)+N_{2} X_{-2}$

$+N_{-2} X_{2}-\left(e^{2 i \omega} X_{2} N_{-2}+e^{-2 i \omega} X_{-2} N_{2}\right)-\left(e^{2 i \omega} X_{2} \Lambda X_{-2}+e^{-2 i \omega} X_{-2} \Lambda X_{2}\right)$.

Take expansions of $T_{n}^{(1)}$ and $\left(T_{n+1}^{(1)}\right)^{-1}$ as $n \rightarrow \infty$ up to the terms of the order $1 / n^{2 \gamma}$. After a long but transparent calculation we have

$$
\begin{aligned}
& \left(T_{n+1}^{(1)}\right)^{-1}\left[\Lambda+\frac{e^{2 i \omega n}}{n^{\gamma}} N_{2}+\frac{e^{-2 i \omega n}}{n^{\gamma}} N_{-2}+R_{n}^{(0)}\right] T_{n}^{(1)} \\
= & \Lambda+\frac{e^{2 i \omega n}}{n^{\gamma}}\left[N_{2}+\Lambda X_{2}-e^{2 i \omega} X_{2} \Lambda\right]+\frac{e^{-2 i \omega n}}{n^{\gamma}}\left[N_{-2}+\Lambda X_{-2}-e^{-2 i \omega} X_{-2} \Lambda\right] \\
+ & \frac{e^{4 i \omega n}}{n^{2 \gamma}}\left[M_{4}+\Lambda X_{4}-e^{4 i \omega} X_{4} \Lambda\right]+\frac{e^{-4 i \omega n}}{n^{2 \gamma}}\left[M_{-4}+\Lambda X_{-4}-e^{-4 i \omega} X_{-4} \Lambda\right] \\
+ & \frac{V}{n^{2 \gamma}}+O\left(\frac{1}{n^{3 \gamma}}+\left|q_{n+1}\right|\right) \quad \text { as } n \rightarrow \infty,
\end{aligned}
$$

since $\left\|R_{n}^{(0)}\right\|=O\left(\left|q_{n+1}\right|\right)$.

We want to cancel the coefficients of $e^{ \pm 2 i \omega n}$ and $e^{ \pm 4 i \omega n}$ in $(3.10)$ by a suitable choice of $X_{ \pm 2}(z)$ and $X_{ \pm 4}(z)$, respectively. To this end, four conditions should be satisfied:

$$
\begin{aligned}
& e^{2 i \omega} X_{2} \Lambda-\Lambda X_{2}=N_{2}, \\
& e^{-2 i \omega} X_{-2} \Lambda-\Lambda X_{-2}=N_{-2}, \\
& e^{4 i \omega} X_{4} \Lambda-\Lambda X_{4}=M_{4}, \\
& e^{-4 i \omega} X_{-4} \Lambda-\Lambda X_{-4}=M_{-4} .
\end{aligned}
$$

We use the following lemma to solve them. 
LEMma 3.2. If $\mu \neq 1, z^{2}, 1 / z^{2}$, then the matrix

$$
X=\left(\begin{array}{cc}
\frac{z f_{11}}{\mu-1} & \frac{z f_{12}}{z^{2} \mu-1} \\
\frac{z f_{21}}{\mu-z^{2}} & \frac{f_{22}}{z(\mu-1)}
\end{array}\right)
$$

satisfies the equation

$$
\mu X \Lambda-\Lambda X=\left(\begin{array}{ll}
f_{11} & f_{12} \\
f_{21} & f_{22}
\end{array}\right) .
$$

Proof. The assertion can be verified by direct substitution.

It follows that we can take

$$
\begin{gathered}
X_{2}(z):=\frac{c e^{i(2 \omega+\delta)}}{2 i\left(z^{2}-1\right)}\left(\begin{array}{cc}
\frac{z}{e^{2 i \omega}-1} & \frac{z^{3}}{z^{2} e^{2 i \omega}-1} \\
-\frac{z}{e^{2 i \omega}-z^{2}} & -\frac{z}{e^{2 i \omega}-1}
\end{array}\right), \\
X_{-2}(z):=-\frac{c e^{-i(2 \omega+\delta)}}{2 i\left(z^{2}-1\right)}\left(\begin{array}{cc}
\frac{z}{e^{-2 i \omega}-1} & \frac{z^{3}}{z^{2} e^{-2 i \omega}-1} \\
-\frac{z}{e^{-2 i \omega}-z^{2}} & -\frac{z}{e^{-2 i \omega}-1}
\end{array}\right)
\end{gathered}
$$

and

$$
X_{ \pm 4}(z):=\left(\begin{array}{cc}
\frac{z\left(M_{ \pm 4}(z)\right)_{11}}{e^{ \pm 4 i \omega}-1} & \frac{z\left(M_{ \pm 4}(z)\right)_{12}}{z^{2} e^{ \pm 4 i \omega}-1} \\
\frac{z\left(M_{ \pm 4}(z)\right)_{21}}{e^{ \pm 4 i \omega}-z^{2}} & \frac{\left(M_{ \pm 4}(z)\right)_{22}}{z\left(e^{ \pm 4 i \omega}-1\right)}
\end{array}\right),
$$

where $\left(M_{ \pm 4}(z)\right)_{11,12,21,22}$ are the entries of the matrix $M_{ \pm 4}(z)$, which are given by (3.8) and (3.11). We see that $X_{ \pm 2}(z)$ are defined and analytic in $\mathbb{C} \backslash\left\{1,-1, e^{ \pm i \omega},-e^{ \pm \imath \omega}\right\}, M_{ \pm 4}(z)$ and $V(z)$ are defined and analytic in $\mathbb{C} \backslash\left\{0,1,-1, e^{ \pm i \omega},-e^{ \pm i \omega}\right\}$, and $X_{ \pm 4}(z), R_{n}^{(2)}(z), T_{n}^{(1)}(z),\left(T_{n}^{(1)}(z)\right)^{-1}$ are defined and analytic in $U$.

With this choice of $T_{n}^{(1)}(z)$ the remainder

$$
\begin{aligned}
R_{n}^{(1)}(z):= & \left(T_{n+1}^{(1)}(z)\right)^{-1}\left[\Lambda(z)+\frac{e^{2 i \omega n}}{n^{\gamma}} N_{2}(z)+\frac{e^{-2 i \omega n}}{n^{\gamma}} N_{-2}(z)+R_{n}^{(0)}(z)\right] T_{n}^{(1)}(z) \\
& -\Lambda(z)-\frac{V(z)}{n^{2 \gamma}}
\end{aligned}
$$

satisfies the estimate

$$
\left\|R_{n}^{(1)}(z)\right\|=O\left(\frac{1}{n^{3 \gamma}}+\left|q_{n+1}\right|\right) \quad \text { as } n \rightarrow \infty
$$

uniformly with respect to $z$ on every compact subset of $U$. 
The second step of the construction of the matrices $T_{n}(z)$ is to consider

$$
T_{n}^{(2)}(z):=\exp \left(\frac{Y(z)}{n^{2 \gamma}}\right)
$$

with some $Y(z)$, which will be determined. Taking the expansions of $T_{n}^{(2)}$ and $\left(T_{n+1}^{(2)}\right)^{-1}$ up to the order of $1 / n^{2 \gamma}$, we have

$$
\begin{aligned}
\left(T_{n+1}^{(2)}\right)^{-1}(\Lambda & \left.+\frac{V}{n^{2 \gamma}}+R_{n}^{(1)}\right) T_{n}^{(2)} \\
= & \Lambda+\frac{1}{n^{2 \gamma}}(V-[Y, \Lambda])+O\left(\frac{1}{n^{3 \gamma}}+\left|q_{n+1}\right|\right) \quad \text { as } n \rightarrow \infty .
\end{aligned}
$$

Let us cancel the anti-diagonal entries of $V-[Y, \Lambda]$ by the choice of $Y$. This leads to the equation

$$
[Y, \Lambda]=\left(\begin{array}{cc}
0 & V_{12} \\
V_{21} & 0
\end{array}\right)
$$

We can take

$$
Y(z):=\frac{z}{z^{2}-1}\left(\begin{array}{cc}
0 & V_{12}(z) \\
-V_{21}(z) & 0
\end{array}\right)
$$

What remains is

$$
\operatorname{diag} V(z)=\mu_{2}(z)\left(\begin{array}{cc}
1 / z & 0 \\
0 & z
\end{array}\right)
$$

which can be seen from (3.9) and (3.11) by a straightforward calculation. The matrices $Y(z), T_{n}^{(2)}(z)$ and $\left(T_{n}^{(2)}(z)\right)^{-1}$ are defined and analytic in $U$. The remainder in the system after the transformation,

$$
R_{n}^{(2)}(z):=\left(T_{n+1}^{(2)}(z)\right)^{-1}\left(\Lambda(z)+\frac{V(z)}{n^{2 \gamma}}+R_{n}^{(1)}(z)\right) T_{n}^{(2)}(z)-\Lambda(z)-\frac{\operatorname{diag} V(z)}{n^{2 \gamma}},
$$

satisfies the estimate

$$
\left\|R_{n}^{(2)}(z)\right\|=O\left(\frac{1}{n^{3 \gamma}}+\left|q_{n+1}\right|\right) \quad \text { as } n \rightarrow \infty
$$

uniformly with respect to $z$ on every compact subset of $U$. Taking $T_{n}(z):=$ $T_{n}^{(1)}(z) T_{n}^{(2)}(z)$ we complete the proof.

Finally, we have come to the following system in L-diagonal form:

$$
\left.w_{n+1}=\left[\begin{array}{cc}
\frac{1}{z}\left(1+\frac{\mu_{2}(z)}{n^{2 \gamma}}\right) & 0 \\
0 & z\left(1-\frac{\mu_{2}(z)}{n^{2 \gamma}}\right)
\end{array}\right)+R_{n}^{(2)}(z)\right] w_{n} .
$$

It is easy to check that for $z \in \mathbb{T} \cap U$ the value $\mu_{2}(z)$ is purely imaginary. 
4. Asymptotic results. In this section we prove several results needed for the analysis of the system (3.12). They are more or less standard, and the approach is similar to [11, 25] and [4. In the cited papers, the existence of a base of solutions with special asymptotic behavior is proven. Here we find the asymptotics of a (roughly speaking) generic solution defined by its initial value.

Let us use the following notation:

$$
\sum_{n=1}^{0}:=0, \quad \prod_{n=1}^{0}:=I,
$$

and for $n_{1}, n_{2} \geq 1$,

$$
\sum_{n=n_{1}}^{n_{2}}:=\sum_{n=1}^{n_{2}}-\sum_{n=1}^{n_{1}-1}, \quad \prod_{n=n_{1}}^{n_{2}}:=\prod_{n=1}^{n_{2}}\left(\prod_{n=1}^{n_{1}-1}\right)^{-1} .
$$

The first lemma is a kind of discrete variation of parameters.

Lemma 4.1. Let $f \in \mathbb{C}^{2}$ and let the matrices $\Lambda_{n}$ be invertible for every $n \geq 1$. If for every $n \geq 1$,

$$
x_{n}=\left(\prod_{l=1}^{n-1} \Lambda_{l}\right) f+\sum_{k=1}^{n-1}\left(\prod_{l=k+1}^{n-1} \Lambda_{l}\right) R_{k} x_{k},
$$

then

$$
x_{n+1}=\left(\Lambda_{n}+R_{n}\right) x_{n}
$$

for every $n \geq 1$.

Proof. Consider

$$
y_{n}^{(1)}:=\left(\prod_{l=1}^{n-1} \Lambda_{l}\right)^{-1} x_{n} .
$$

We can rewrite 4.2 as

$$
y_{n+1}^{(1)}-y_{n}^{(1)}=\left(\prod_{l=1}^{n} \Lambda_{l}\right)^{-1} R_{n} x_{n} .
$$

At the same time 4.1) is equivalent to

$$
y_{n}^{(1)}=f+\sum_{k=1}^{n-1}\left(\prod_{l=1}^{k} \Lambda_{l}\right)^{-1} R_{k} x_{k} .
$$

Clearly, 4.3 follows from 4.4).

REMARK 4.2. Lemma 4.1 says that the $x$ given by 4.1 is the solution of the system 4.2 with $x_{1}=f$. Every solution $x$ of 4.2 can be represented in the form 4.1) with $f:=x_{1}$. 
In what follows let us consider systems with matrices $\Lambda_{n}$ of the form

$$
\Lambda_{n}:=\left(\begin{array}{cc}
\lambda_{n} & 0 \\
0 & 1 / \lambda_{n}
\end{array}\right),
$$

where $\lambda_{n} \in \mathbb{C}$. The following lemma gives an estimate on the growth of solutions of the system 4.2 .

Lemma 4.3. Suppose that

$$
\sum_{k=1}^{\infty} \frac{\left\|R_{k}\right\|}{\left|\lambda_{k}\right|}<\infty
$$

and there exists $M$ such that for every $m \geq n$,

$$
\prod_{l=n+1}^{m}\left|\lambda_{l}\right| \geq \frac{1}{M}
$$

Then every solution $x$ of the system

$$
x_{n+1}=\left[\left(\begin{array}{cc}
\lambda_{n} & 0 \\
0 & 1 / \lambda_{n}
\end{array}\right)+R_{n}\right] x_{n}, \quad n \geq 1,
$$

satisfies the estimate

$$
\left\|x_{n}\right\| \leq\left(\prod_{l=1}^{n-1}\left|\lambda_{l}\right|\right) \exp \left(\left(1+M^{2}\right) \sum_{k=1}^{\infty} \frac{\left\|R_{k}\right\|}{\left|\lambda_{k}\right|}\right)\left(1+M^{2}\right)\left\|x_{1}\right\| .
$$

Proof. Let

$$
f=\left(\begin{array}{l}
f_{1} \\
f_{2}
\end{array}\right):=x_{1}, \quad y_{n}^{(2)}:=\frac{x_{n}}{\prod_{l=1}^{n-1} \lambda_{l}} .
$$

Then (by Lemma 4.1$\}\left\{y_{n}^{(2)}\right\}$ satisfies the equation

$$
y_{n}^{(2)}=\left(\begin{array}{cc}
1 & 0 \\
0 & \prod_{l=1}^{n-1} \lambda_{l}^{-2}
\end{array}\right) f+\sum_{k=1}^{n-1}\left(\begin{array}{cc}
1 & 0 \\
0 & \prod_{l=k+1}^{n-1} \lambda_{l}^{-2}
\end{array}\right) \frac{R_{k}}{\lambda_{k}} y_{k}^{(2)} .
$$

Consider this as an equation in the Banach space $l^{\infty}\left(\mathbb{C}^{2}\right)$. Consider the vector

$$
\hat{f}:=\left\{\left(\begin{array}{c}
f_{1} \\
\left(\prod_{l=1}^{n-1} \lambda_{l}^{-2}\right) f_{2}
\end{array}\right)\right\}_{n=1}^{\infty}
$$

and the operator

$$
V:\left\{u_{n}\right\}_{n=1}^{\infty} \mapsto\left\{\sum_{k=1}^{n-1}\left(\begin{array}{cc}
1 & 0 \\
0 & \prod_{l=k+1}^{n-1} \lambda_{l}^{-2}
\end{array}\right) \frac{R_{k}}{\lambda_{k}} u_{k}\right\}_{n=1}^{\infty} .
$$

In this notation (4.7) reads

$$
y=\hat{f}+V y .
$$


The powers of the operator $V$ can be estimated as follows:

$$
\left\|V^{m}\right\|_{l^{\infty}} \leq \frac{\left(\left(1+M^{2}\right) \sum_{k=1}^{\infty}\left\|R_{k}\right\| /\left|\lambda_{k}\right|\right)^{m}}{m !},
$$

and so $(I-V)^{-1}$ exists and

$$
\left\|(I-V)^{-1}\right\|_{l^{\infty}} \leq \exp \left(\left(1+M^{2}\right) \sum_{k=1}^{\infty} \frac{\left\|R_{k}\right\|}{\left|\lambda_{k}\right|}\right) .
$$

Therefore

$$
\left\|y^{(2)}\right\|_{l^{\infty}} \leq \exp \left(\left(1+M^{2}\right) \sum_{k=1}^{\infty} \frac{\left\|R_{k}\right\|}{\left|\lambda_{k}\right|}\right)\left(1+M^{2}\right)\|f\| .
$$

Returning to the solution $x$ we arrive at the desired estimate 4.6.

The following lemma gives the asymptotics of the solutions of the system 4.5.

Lemma 4.4. Suppose that

$$
\sum_{k=1}^{\infty} \frac{\left\|R_{k}\right\|}{\left|\lambda_{k}\right|}<\infty
$$

and there exists $M$ such that for every $m \geq n$,

$$
\prod_{l=n+1}^{m}\left|\lambda_{l}\right| \geq \frac{1}{M}
$$

Suppose that $x$ is a solution of the system

$$
x_{n+1}=\left[\left(\begin{array}{cc}
\lambda_{n} & 0 \\
0 & 1 / \lambda_{n}
\end{array}\right)+R_{n}\right] x_{n}, \quad n \geq 1 .
$$

(a) If

$$
\prod_{l=1}^{\infty}\left|\lambda_{l}\right|<\infty
$$

then

$$
\lim _{n \rightarrow \infty}\left(\prod_{l=1}^{n-1} \Lambda_{l}\right)^{-1} x_{n}=x_{1}+\sum_{k=1}^{\infty}\left(\prod_{l=1}^{k} \Lambda_{l}\right)^{-1} R_{k} x_{k}
$$

(the limit and the sum both exist and the equality holds).

(b) If

$$
\prod_{l=1}^{\infty} \lambda_{l}=\infty
$$


then

$$
\lim _{n \rightarrow \infty} \frac{x_{n}}{\prod_{l=1}^{n-1} \lambda_{l}}=\left(\begin{array}{ll}
1 & 0 \\
0 & 0
\end{array}\right)\left[x_{1}+\sum_{k=1}^{\infty} \frac{R_{k} x_{k}}{\prod_{l=1}^{k} \lambda_{l}}\right]
$$

(the limit and the sum both exist and the equality holds).

Proof. (a) Equation (4.4) can be rewritten as follows:

$$
\left(\prod_{l=1}^{n-1} \Lambda_{l}\right)^{-1} x_{n}=x_{1}+\sum_{k=1}^{n-1}\left(\prod_{l=1}^{k} \Lambda_{l}\right)^{-1} R_{k} x_{k} .
$$

Let us show that the sum on the right-hand side is convergent. By Lemma 4.3 .

$$
\begin{aligned}
\left\|\left(\prod_{l=1}^{k} \Lambda_{l}\right)^{-1} R_{k} x_{k}\right\| \leq & \frac{\left\|R_{k}\right\|}{\left|\lambda_{k}\right|}\left\|\left(\prod_{l=1}^{k} \frac{\Lambda_{l}}{\lambda_{l}}\right)^{-1}\right\| \\
& \times \exp \left(\left(1+M^{2}\right) \sum_{m=1}^{\infty} \frac{\left\|R_{m}\right\|}{\left|\lambda_{m}\right|}\right)\left(1+M^{2}\right)\left\|x_{1}\right\| .
\end{aligned}
$$

Since

$$
\left\|\left(\prod_{l=1}^{k} \frac{\Lambda_{l}}{\lambda_{l}}\right)^{-1}\right\|=\left\|\left(\begin{array}{cc}
1 & 0 \\
0 & \prod_{l=1}^{k} \lambda_{l}^{2}
\end{array}\right)\right\| \leq \sqrt{1+\left(\prod_{l=1}^{k}\left|\lambda_{l}\right|\right)^{2}}
$$

is bounded by hypothesis, we have

$$
\left\|\left(\prod_{l=1}^{k} \Lambda_{l}\right)^{-1} R_{k} x_{k}\right\| \leq \mathrm{const} \cdot \frac{\left\|R_{k}\right\|}{\left|\lambda_{k}\right|}
$$

which is summable. Therefore the limit of $(4.12)$ as $n \rightarrow \infty$ exists.

(b) Consider the sum on the right-hand side of (4.7). Lemma 4.3 yields

$$
\begin{aligned}
\left\|\left(\begin{array}{cc}
1 & 0 \\
0 & \prod_{l=k+1}^{n-1} \lambda_{l}^{-2}
\end{array}\right) \frac{R_{k}}{\lambda_{k}} y_{k}^{(2)}\right\| & \leq\left(1+M^{2}\right) \frac{\left\|R_{k}\right\|}{\left|\lambda_{k}\right|}\left\|y_{k}^{(2)}\right\| \\
\leq & \frac{\left\|R_{k}\right\|}{\left|\lambda_{k}\right|}\left(1+M^{2}\right)^{2} \exp \left(\left(1+M^{2}\right) \sum_{m=1}^{\infty} \frac{\left\|R_{m}\right\|}{\left|\lambda_{m}\right|}\right)\left\|x_{1}\right\|,
\end{aligned}
$$

which is summable. Since 4.11 holds, by Lebesgue's dominated convergence theorem the limit as $n \rightarrow \infty$ of 4.7 exists:

$$
\lim _{n \rightarrow \infty} y_{n}^{(2)}=\left(\begin{array}{ll}
1 & 0 \\
0 & 0
\end{array}\right) x_{1}+\sum_{k=1}^{\infty}\left(\begin{array}{ll}
1 & 0 \\
0 & 0
\end{array}\right) \frac{R_{k}}{\lambda_{k}} y_{k}^{(2)} .
$$

Returning to $x$ from $y^{(2)}$ we obtain the assertion of the lemma in case (b).

REMARK 4.5. Condition (4.8) together with 4.10) or 4.11) is a case of the standard dichotomy (Levinson) condition (cf. [4], [11], [25], [26]). 
5. Asymptotics of polynomials, the Jost function and the spectral density. In this section we apply the results of the previous two sections to find the asymptotics of the polynomials $P_{n}(\lambda)$ associated to the matrix $\mathcal{J}$ and to prove the Weyl-Titchmarsh type formula for the spectral density.

THEOREM 5.1. Let $\left\{b_{n}\right\}$ be given by (1.1) and suppose the condition (1.2) holds. Let $P_{n}(\lambda)$ be the orthonormal polynomials associated to the Jacobi matrix $\mathcal{J}$ given by $(1.3)$. Then for every $z \in \overline{\mathbb{D}} \cap U$ (where $U$ is given by (3.4) there exists $F(z)$ (the Jost function) such that:

- if $z \in \mathbb{D} \backslash\{0\}$ (i.e., $\lambda=z+1 / z \in \mathbb{C} \backslash[-2 ; 2]$ ), then

$$
\begin{aligned}
P_{n}\left(z+\frac{1}{z}\right)= & \frac{z F(z)}{1-z^{2}} \frac{1}{z^{n}} \exp \left(\frac{\mu_{2}(z) n^{1-2 \gamma}}{1-2 \gamma}\right) \\
& +o\left(\frac{1}{|z|^{n}} \exp \left(\frac{\operatorname{Re} \mu_{2}(z) n^{1-2 \gamma}}{1-2 \gamma}\right)\right) \quad \text { as } n \rightarrow \infty
\end{aligned}
$$

(where $\mu_{2}(z)$ is given by 1.5$)$ ),

- if $z \in \mathbb{T} \cap U$ (i.e., $\lambda=z+1 / z \in(-2 ; 2) \backslash\{ \pm 2 \cos \omega, \pm 2 \cos 2 \omega\})$, then

$$
\begin{aligned}
P_{n}\left(z+\frac{1}{z}\right)= & \frac{z F(z)}{1-z^{2}} \frac{1}{z^{n}} \exp \left(\frac{\mu_{2}(z) n^{1-2 \gamma}}{1-2 \gamma}\right) \\
& +\frac{z \overline{F(z)}}{z^{2}-1} z^{n} \exp \left(-\frac{\mu_{2}(z) n^{1-2 \gamma}}{1-2 \gamma}\right)+o(1) \quad \text { as } n \rightarrow \infty .
\end{aligned}
$$

The function $F$ is analytic in $\mathbb{D} \backslash\{0\}$ and continuous in $\mathbb{T} \cap U$.

Proof. For $z \in U$ every solution $u$ of the spectral equation (3.1) corresponds to a solution $w$ of 3.12 by the equality

$$
\left(\begin{array}{c}
u_{n} \\
u_{n+1}
\end{array}\right)=\left(\begin{array}{cc}
1 & 1 \\
1 / z & z
\end{array}\right) T_{n}(z) w_{n}
$$

Let us define the solution $\varphi(z)$ of $(3.12)$ that corresponds to the polynomials $P_{n}(\lambda)$ :

$$
\varphi_{n}(z):=T_{n}^{-1}(z)\left(\begin{array}{cc}
1 & 1 \\
1 / z & z
\end{array}\right)^{-1}\left(\begin{array}{c}
P_{n}(z+1 / z) \\
P_{n+1}(z+1 / z)
\end{array}\right) .
$$

Define

$$
\lambda_{n}(z):= \begin{cases}\frac{1}{z} \exp \left(\frac{\mu_{2}(z)}{1-2 \gamma}\left((n+1)^{1-2 \gamma}-n^{1-2 \gamma}\right)\right) & \text { if } n \geq 2, \\ \frac{1}{z^{2}} \exp \left(\frac{\mu_{2}(z)}{1-2 \gamma} 2^{1-2 \gamma}\right) & \text { if } n=1,\end{cases}
$$


and

$$
R_{n}^{(3)}(z):=R_{n}^{(2)}(z)+\left(\begin{array}{cc}
\frac{1}{z}\left(1+\frac{\mu_{2}(z)}{n^{2 \gamma}}\right)-\lambda_{n}(z) & 0 \\
0 & z\left(1-\frac{\mu_{2}(z)}{n^{2 \gamma}}\right)-\frac{1}{\lambda_{n}(z)}
\end{array}\right),
$$

so that the system $(3.12)$ reads

$$
w_{n+1}=\left[\left(\begin{array}{cc}
\lambda_{n}(z) & 0 \\
0 & 1 / \lambda_{n}(z)
\end{array}\right)+R_{n}^{(3)}(z)\right] w_{n}, \quad n \geq 2 .
$$

Let us check that Lemmas 4.3 and 4.4 are applicable to this system. With the definition (5.4), the product of the diagonal entries looks simple:

$$
\prod_{l=1}^{n-1} \lambda_{l}(z)=\frac{1}{z^{n}} \exp \left(\frac{\mu_{2}(z) n^{1-2 \gamma}}{1-2 \gamma}\right), \quad n \geq 2 .
$$

Let $K$ be a compact subset of $\overline{\mathbb{D}} \cap U$. For $m \geq n$ we have

$$
\begin{aligned}
\prod_{l=n+1}^{m}\left|\lambda_{l}(z)\right| & =\frac{1}{|z|^{m-n}} \exp \left(\frac{\operatorname{Re} \mu_{2}(z)}{1-2 \gamma}\left((m+1)^{1-2 \gamma}-(n+1)^{1-2 \gamma}\right)\right) \\
& \geq \frac{1}{|z|^{m-n}} \exp \left(-\frac{\left|\operatorname{Re} \mu_{2}(z)\right|}{1-2 \gamma}(m-n)^{1-2 \gamma}\right),
\end{aligned}
$$

where we have used the inequality

$$
(m+1)^{1-2 \gamma}-(n+1)^{1-2 \gamma} \leq(m-n)^{1-2 \gamma}
$$

(which holds because the function $x \mapsto x^{1-2 \gamma}$ is concave). Further, since $\operatorname{Re} \mu_{2}(z)=0$ for $z \in \mathbb{T}$, the function

$$
z \mapsto \frac{\operatorname{Re} \mu_{2}(z)}{1-|z|}
$$

is smooth on $U$, hence there exists $c_{1}(K)$ such that for every $z \in K$,

$$
\left|\operatorname{Re} \mu_{2}(z)\right| \leq c_{1}(K)(1-|z|) .
$$

Also $|z| \leq e^{|z|-1}$, for every $z$ and therefore

$$
\prod_{l=n+1}^{m}\left|\lambda_{l}(z)\right| \geq \exp \left[(1-|z|)\left(m-n-\frac{c_{1}(K)}{1-2 \gamma}(m-n)^{1-2 \gamma}\right)\right] .
$$

Let

$$
c_{2}(K):=\sup _{x \geq 0}\left(\frac{c_{1}(K)}{1-2 \gamma} x^{1-2 \gamma}-x\right),
$$

which is finite. For every $z \in K$ and $m \geq n$, we have

$$
(1-|z|)\left(m-n-\frac{c_{1}(K)}{1-2 \gamma}(m-n)^{1-2 \gamma}\right) \in\left[-c_{2}(K) ;+\infty\right)
$$


and

Further,

$$
\prod_{l=n+1}^{m}\left|\lambda_{l}(z)\right| \geq e^{-c_{2}(K)}
$$

$$
\left\|R_{n}^{(2)}(z)\right\|=O\left(\frac{1}{n^{3 \gamma}}+\left|q_{n+1}\right|\right) \quad \text { as } n \rightarrow \infty
$$

uniformly with respect to $z \in K$ and

$$
\begin{aligned}
\frac{1}{z}\left(1+\frac{\mu_{2}(z)}{n^{2 \gamma}}\right) & -\lambda_{n}(z) \\
= & \frac{1}{z}\left(1+\frac{\mu_{2}(z)}{n^{2 \gamma}}-\exp \left[\frac{\mu_{2}(z)}{1-2 \gamma}\left((n+1)^{1-2 \gamma}-n^{1-2 \gamma}\right)\right]\right) \\
= & O\left(\frac{1}{n^{2 \gamma+1}}\right) \quad \text { as } n \rightarrow \infty .
\end{aligned}
$$

Analogously

and finally

$$
z\left(1-\frac{\mu_{2}(z)}{n^{2 \gamma}}\right)-\frac{1}{\lambda_{n}(z)}=O\left(\frac{1}{n^{2 \gamma+1}}\right)
$$

$$
\left\|R_{n}^{(3)}(z)\right\|=O\left(\frac{1}{n^{3 \gamma}}+\left|q_{n+1}\right|\right) \quad \text { as } n \rightarrow \infty
$$

uniformly with respect to $z \in K$. Also for every $z \in K$ and $n$,

$$
\frac{1}{\left|\lambda_{n}(z)\right|} \leq|z| \exp \left(\frac{2\left|\operatorname{Re} \mu_{2}(z)\right|}{1-2 \gamma}\right) \text {. }
$$

Thus the sum

$$
\sum_{n=1}^{\infty} \frac{\left\|R_{n}^{(3)}(z)\right\|}{\left|\lambda_{n}(z)\right|}
$$

as a function of $z$ is bounded on $K$ (in fact it is continuous in $U$ ). For $z \in \mathbb{T} \cap K, \prod_{l=1}^{n} \lambda_{l}(z)$ is bounded, while for $z \in \mathbb{D} \cap K$,

$$
\prod_{l=1}^{n} \lambda_{l}(z) \rightarrow \infty \quad \text { as } n \rightarrow \infty .
$$

We see that Lemma 4.4 is applicable. It implies that for every $z \in K$ we can define

$$
\Phi(z):=\left(\varphi_{1}(z)+\sum_{n=1}^{\infty} z^{n} \exp \left(-\frac{\mu_{2}(z) n^{1-2 \gamma}}{1-2 \gamma}\right) R_{n}^{(3)}(z) \varphi_{n}(z)\right)_{1}
$$

for every $z \in \mathbb{T} \cap K$ we can define

$$
\widetilde{\Phi}(z):=\left(\varphi_{1}(z)+\sum_{n=1}^{\infty} \frac{1}{z^{n}} \exp \left(\frac{\mu_{2}(z) n^{1-2 \gamma}}{1-2 \gamma}\right) R_{n}^{(3)}(z) \varphi_{n}(z)\right)_{2},
$$

and the solution $\varphi(z)$ has the following asymptotics: for $z \in \mathbb{T} \cap K$ (case (a) 
of Lemma 4.4,

$$
\left.\varphi_{n}(z)=\left(\begin{array}{cc}
\frac{1}{z^{n}} \exp \left(\frac{\mu_{2}(z) n^{1-2 \gamma}}{1-2 \gamma}\right) & 0 \\
0 & z^{n} \exp \left(-\frac{\mu_{2}(z) n^{1-2 \gamma}}{1-2 \gamma}\right)
\end{array}\right)\left(\begin{array}{l}
\Phi(z) \\
\widetilde{\Phi}(z)
\end{array}\right)+o(1)\right)
$$

as $n \rightarrow \infty$, and for $z \in \mathbb{D} \cap K$ (case (b) of Lemma 4.4),

$$
\varphi_{n}(z)=\frac{1}{z^{n}} \exp \left(\frac{\mu_{2}(z) n^{1-2 \gamma}}{1-2 \gamma}\right)\left(\left(\begin{array}{c}
\Phi(z) \\
0
\end{array}\right)+o(1)\right) \quad \text { as } n \rightarrow \infty .
$$

We can see Lemma 4.3 is also applicable. Let

$$
\begin{aligned}
& c_{3}(K):=\max _{z \in K} \sum_{n=1}^{\infty} \frac{\left\|R_{n}^{(3)}(z)\right\|}{\left|\lambda_{n}(z)\right|} \\
& c_{4}(K):=\exp \left(\left(1+e^{2 c_{2}(K)}\right) c_{3}(K)\right)\left(1+e^{2 c_{2}(K)}\right) \max _{z \in K}\left\|\varphi_{1}(z)\right\| .
\end{aligned}
$$

Lemma 4.3 implies that for every $z \in K$ and $n$,

$$
\left\|\varphi_{n}(z)\right\| \leq \frac{c_{4}(K)}{|z|^{n}} \exp \left(\frac{\operatorname{Re} \mu_{2}(z) n^{1-2 \gamma}}{1-2 \gamma}\right) .
$$

Consider the expression $(5.6)$ for $\Phi(z)$. We have

$$
\begin{aligned}
\left\|z^{n} \exp \left(-\frac{\mu_{2}(z) n^{1-2 \gamma}}{1-2 \gamma}\right) R_{n}^{(3)}(z) \varphi_{n}(z)\right\| & \leq c_{4}(K)\left\|R_{n}^{(3)}(z)\right\| \\
& =O\left(\frac{1}{n^{3 \gamma}}+\left|q_{n+1}\right|\right) \quad \text { as } n \rightarrow \infty
\end{aligned}
$$

uniformly with respect to $z \in K$. It follows that the function $\Phi$ is analytic in the interior of $K$ and continuous in $K$. Since the set $K \subset \overline{\mathbb{D}} \cap U$ is arbitrary, $\Phi$ exists and is continuous in $\overline{\mathbb{D}} \cap U$ and is analytic in $\mathbb{D} \backslash\{0\}$. The asymptotics (5.7) holds for every $z \in \mathbb{T} \cap U$ and the asymptotics (5.8) holds for every $z \in \mathbb{D} \backslash\{0\}$.

It follows that for $z \in \mathbb{D} \backslash\{0\}$,

$$
\begin{aligned}
\left(\begin{array}{c}
P_{n}(z+1 / z) \\
P_{n+1}(z+1 / z)
\end{array}\right) & =\frac{1}{z^{n}} \exp \left(\frac{\mu_{2}(z) n^{1-2 \gamma}}{1-2 \gamma}\right)\left(\begin{array}{cc}
1 & 1 \\
1 / z & z
\end{array}\right) T_{n}(z)\left(\left(\begin{array}{c}
\Phi(z) \\
0
\end{array}\right)+o(1)\right) \\
& =\Phi(z) \frac{1}{z^{n}} \exp \left(\frac{\mu_{2}(z) n^{1-2 \gamma}}{1-2 \gamma}\right)\left(\left(\begin{array}{c}
1 \\
1 / z
\end{array}\right)+o(1)\right)
\end{aligned}
$$

as $n \rightarrow \infty$, since $T_{n}(z)=I+o(1)$. If we define

$$
F(z):=\Phi(z) \frac{1-z^{2}}{z}
$$

then we arrive at the first assertion of the theorem. 
It also follows in an analogous fashion that for $z \in \mathbb{T} \cap U$,

$$
\begin{aligned}
\left(\begin{array}{c}
P_{n}(z+1 / z) \\
P_{n+1}(z+1 / z)
\end{array}\right)= & \Phi(z) \frac{1}{z^{n}} \exp \left(\frac{\mu_{2}(z) n^{1-2 \gamma}}{1-2 \gamma}\right)\left(\begin{array}{c}
1 \\
1 / z
\end{array}\right) \\
& +\widetilde{\Phi}(z) z^{n} \exp \left(-\frac{\mu_{2}(z) n^{1-2 \gamma}}{1-2 \gamma}\right)\left(\begin{array}{l}
1 \\
z
\end{array}\right)+o(1)
\end{aligned}
$$

as $n \rightarrow \infty$. The first component of this vector equality gives the asymptotics of $P_{n}(z+1 / z)$, and to complete the proof we only need the following lemma.

Lemma 5.2. For every $z \in \mathbb{T} \cap U$,

$$
\widetilde{\Phi}(z)=\overline{\Phi(z)} .
$$

Proof. This follows from the fact that the values of the polynomials $P_{n}(z+1 / z)$ for $z \in \mathbb{T}$ are real. Consider the imaginary part of the first component of (5.10):

$$
\begin{aligned}
0= & \frac{\Phi(z)-\overline{\widetilde{\Phi}(z)}}{2 i} \frac{1}{z^{n}} \exp \left(\frac{\mu_{2}(z) n^{1-2 \gamma}}{1-2 \gamma}\right) \\
& +\frac{\widetilde{\Phi}(z)-\overline{\Phi(z)}}{2 i} z^{n} \exp \left(-\frac{\mu_{2}(z) n^{1-2 \gamma}}{1-2 \gamma}\right)+o(1) \quad \text { as } n \rightarrow \infty .
\end{aligned}
$$

Suppose that $\widetilde{\Phi}(z) \neq \overline{\Phi(z)}$. Then

$$
z^{2 n} \exp \left(2 i \arg (\overline{\Phi(z)}-\widetilde{\Phi}(z))-\frac{2 \mu_{2}(z)}{1-2 \gamma} n^{1-2 \gamma}\right) \rightarrow 1 \quad \text { as } n \rightarrow \infty .
$$

Let

$$
\hat{\Phi}(z):=\exp [2 i \arg (\overline{\Phi(z)}-\widetilde{\Phi}(z))], \quad \hat{\mu}(z):=-\frac{2 \mu_{2}(z)}{1-2 \gamma} .
$$

We have

$$
\hat{\Phi}(z) z^{2 n} e^{\hat{\mu}(z) n^{1-2 \gamma}} \rightarrow 1 \quad \text { as } n \rightarrow \infty .
$$

On the other hand,

$$
\hat{\Phi}(z) z^{2(n+1)} e^{\hat{\mu}(z)(n+1)^{1-2 \gamma}}=\hat{\Phi}(z) z^{2 n} e^{\hat{\mu}(z) n^{1-2 \gamma}} z^{2}\left(1+O\left(\frac{1}{n^{2 \gamma}}\right)\right) \rightarrow z^{2} .
$$

It follows that $z^{2}=1$, which is a contradiction.

This completes the proof of the theorem.

The following (final) theorem gives a formula of the Weyl-Titchmarsh (or Kodaira) type for the spectral density. It follows from the asymptotics of the orthogonal polynomials given by Theorem 5.1 in a standard way (see [27. Chapter 5] and [16]) and contains the Jost function, which appears in the asymptotics of $P_{n}(\lambda)$. 
THEOREM 5.3. Let $\left\{b_{n}\right\}$ be given by (1.1) and suppose the condition (1.2) holds. Then the spectrum of the Jacobi matrix $\mathcal{J}$ given by 1.3 is purely absolutely continuous on $(-2 ; 2) \backslash\{ \pm 2 \cos \omega, \pm 2 \cos 2 \omega\}$, and for a.a. $\lambda \in(-2 ; 2)$ the spectral density of $\mathcal{J}$ equals

$$
\rho^{\prime}(\lambda)=\frac{\sqrt{4-\lambda^{2}}}{2 \pi\left|F\left(\frac{\lambda}{2}-i \frac{\sqrt{4-\lambda^{2}}}{2}\right)\right|^{2}}
$$

(the Weyl-Titchmarsh type formula), where the Jost function $F$ is defined in Theorem 5.1. The denominator in (5.11) does not vanish for $\lambda \in(-2 ; 2) \backslash$ $\{ \pm 2 \cos \omega, \pm 2 \cos 2 \omega\}$.

Proof. If we rewrite 5.11 in terms of the variable $z$, it reads

$$
\rho^{\prime}\left(z+\frac{1}{z}\right)=\frac{1-z^{2}}{2 \pi i z|F(z)|^{2}} .
$$

Polynomials of the second kind have asymptotics of the same type as polynomials of the first kind. Indeed, define the truncated Jacobi matrix $\mathcal{J}_{1}$ to be the original matrix $\mathcal{J}$ with the first row and the first column removed,

$$
\mathcal{J}_{1}=\left(\begin{array}{cccc}
b_{2} & 1 & 0 & \cdots \\
1 & b_{3} & 1 & \cdots \\
0 & 1 & b_{4} & \cdots \\
\vdots & \vdots & \vdots & \ddots
\end{array}\right)
$$

The polynomials of the second kind $Q_{n}(\lambda)$ associated to $\mathcal{J}$ are the polynomials of the first kind for $\mathcal{J}_{1}$. The matrix $\mathcal{J}_{1}$ also satisfies the conditions of Theorem 5.1, which shows that there exists a function $F_{1}$ analytic in $\mathbb{D} \backslash\{0\}$ and continuous in $\overline{\mathbb{D}} \cap U$ such that for $z \in \mathbb{D} \backslash\{0\}$,

$$
\begin{aligned}
Q_{n}\left(z+\frac{1}{z}\right)= & \frac{z F_{1}(z)}{1-z^{2}} \frac{1}{z^{n}} \exp \left(\frac{\mu_{2}(z) n^{1-2 \gamma}}{1-2 \gamma}\right) \\
& +o\left(\frac{1}{|z|^{n}} \exp \left(\frac{\operatorname{Re} \mu_{2}(z) n^{1-2 \gamma}}{1-2 \gamma}\right)\right) \quad \text { as } n \rightarrow \infty .
\end{aligned}
$$

This and the asymptotics (5.1) of $P_{n}$ imply that the combination $Q_{n}(\lambda)+$ $m(\lambda) P_{n}(\lambda)$ belongs to $l^{2}$ for $\lambda \in \mathbb{C}_{+}$and $\lambda \in \mathbb{C}_{-}$only if

$$
m\left(z+\frac{1}{z}\right)=-\frac{F_{1}(z)}{F(z)} \quad \text { for } z \in \mathbb{D} \backslash(-1 ; 1) .
$$

It follows that the zeros of $F$ in $\mathbb{D}$ correspond to the eigenvalues of $\mathcal{J}$ outside the interval $[-2 ; 2]$ and hence can only lie in $(-1 ; 1)$. For every $z \in \mathbb{T} \cap U$, 5.14 has a limit,

$$
m\left(z+\frac{1}{z}+i 0\right)=-\frac{F_{1}(z)}{F(z)}
$$


This is what we are looking for, because

$$
\rho^{\prime}\left(z+\frac{1}{z}\right)=\frac{1}{\pi} \operatorname{Re} m\left(z+\frac{1}{z}+i 0\right)=\frac{F(z) \overline{F_{1}(z)}-\overline{F(z)} F_{1}(z)}{2 \pi i|F(z)|^{2}},
$$

and the spectrum of $\mathcal{J}$ is purely absolutely continuous on the intervals $(-2 ; 2) \backslash\{ \pm 2 \cos \omega, \pm 2 \cos 2 \omega\}$ (from the fact that the limit is finite at every point of these intervals, [8], [14]).

Theorem 5.1 also gives, for $z \in \mathbb{T} \cap U$,

$$
\begin{aligned}
Q_{n}\left(z+\frac{1}{z}\right) & =\frac{z F_{1}(z)}{1-z^{2}} \frac{1}{z^{n}} \exp \left(\frac{\mu_{2}(z) n^{1-2 \gamma}}{1-2 \gamma}\right) \\
& +\frac{z \overline{F_{1}(z)}}{z^{2}-1} z^{n} \exp \left(-\frac{\mu_{2}(z) n^{1-2 \gamma}}{1-2 \gamma}\right)+o(1) \quad \text { as } n \rightarrow \infty
\end{aligned}
$$

Substituting this expression and the analogous one (5.2) for $P_{n}$ into the formula for the Wronskian of $P$ and $Q$ (which is constant and equal to one), after a short calculation we get

$$
\begin{aligned}
1 & =W\left(P\left(z+\frac{1}{z}\right), Q\left(z+\frac{1}{z}\right)\right) \\
& =P_{n}\left(z+\frac{1}{z}\right) Q_{n+1}\left(z+\frac{1}{z}\right)-P_{n+1}\left(z+\frac{1}{z}\right) Q_{n}\left(z+\frac{1}{z}\right) \\
& =\frac{z\left(F(z) \overline{F_{1}(z)}-\overline{F(z)} F_{1}(z)\right)}{1-z^{2}},
\end{aligned}
$$

where the $o(1)$ terms cancel each other, so that the result does not depend on $n$. From this we have

$$
F(z) \overline{F_{1}(z)}-\overline{F(z)} F_{1}(z)=\frac{1}{z}-z,
$$

which together with 5.12 gives 5.11 .

COROLlary 5.4. Under the conditions of Theorem 5.3,

$$
\rho^{\prime}(\lambda)=\frac{\sqrt{4-\lambda^{2}}}{2 \pi \lim _{n \rightarrow \infty}\left|P_{n+1}(\lambda)-z P_{n}(\lambda)\right|^{2}} \quad \text { for a.a. } \lambda \in(-2 ; 2) .
$$

Proof. From (5.3), 5.7), Lemma 5.2 and (5.9) we have: for $z \in \mathbb{T} \cap U$,

$$
\begin{array}{r}
\left(\begin{array}{r}
\left(P_{n+1}\left(z+\frac{1}{z}\right)-z P_{n}\left(z+\frac{1}{z}\right)\right) z^{n} \exp \left(-\frac{\mu_{2}(z) n^{1-2 \gamma}}{1-2 \gamma}\right) \\
\left(P_{n+1}\left(z+\frac{1}{z}\right)-\frac{1}{z} P_{n}\left(z+\frac{1}{z}\right)\right)
\end{array}\right) \\
=\left(\frac{F(z)}{z^{n}} \exp \left(\frac{\mu_{2}(z) n^{1-2 \gamma}}{1-2 \gamma}\right)+o(1) \quad \text { as } n \rightarrow \infty .\right.
\end{array}
$$


Therefore

$$
\left|P_{n+1}(\lambda)-z P_{n}(\lambda)\right| \rightarrow|F(z)| \quad \text { as } n \rightarrow \infty,
$$

and together with (5.11) we obtain the assertion of the corollary.

6. The case $\gamma \in(1 / 2 ; 1]$. In this section we formulate the result for the simpler case $\gamma \in(1 / 2 ; 1]$ and show how to simplify and modify the proof of the corresponding result for $\gamma \in(1 / 3 ; 1 / 2)$ to fit this case. We will need this as a step in proving the asymptotics of the spectral density (1.7).

Theorem 6.1. Let $\left\{b_{n}\right\}_{n=1}^{\infty}$ be given by (1.1) and

$$
\gamma \in(1 / 2 ; 1], \quad \omega \notin \pi \mathbb{Z}, \quad\left\{q_{n}\right\}_{n=1}^{\infty} \in l^{1} .
$$

Then for every $z \in \mathbb{T} \backslash\left\{1,-1, e^{ \pm i \omega},-e^{ \pm i \omega}\right\}$ there exists $F(z)$ such that the orthonormal polynomials $P_{n}$ associated to $\left\{b_{n}\right\}_{n=1}^{\infty}$ have the following asymptotics:

$$
P_{n}\left(z+\frac{1}{z}\right)=\frac{z F(z)}{1-z^{2}} \frac{1}{z^{n}}+\frac{z \overline{F(z)}}{z^{2}-1} z^{n}+o(1) \quad \text { as } n \rightarrow \infty .
$$

The function $F$ is continuous in $\mathbb{T} \backslash\left\{1,-1, e^{ \pm i \omega},-e^{ \pm i \omega}\right\}$ and does not have zeros there. The spectrum of the Jacobi matrix $\mathcal{J}$ given by $(1.3)$ is purely absolutely continuous on $(-2 ; 2) \backslash\{ \pm 2 \cos \omega\}$, and for a.a. $\lambda \in(-2 ; 2)$ the spectral density of $\mathcal{J}$ equals

$$
\rho^{\prime}(\lambda)=\frac{\sqrt{4-\lambda^{2}}}{2 \pi\left|F\left(\frac{\lambda}{2}-i \frac{\sqrt{4-\lambda^{2}}}{2}\right)\right|^{2}} .
$$

Proof. We assume $\gamma \in(1 / 2 ; 1]$ and return to previous sections. The statement of Lemma 3.1 holds true if we replace $U$ with

$$
\mathbb{C} \backslash\left\{0,1,-1, e^{ \pm \omega},-e^{ \pm \omega}\right\},
$$

the estimate (3.5) with

$$
\left\|R_{n}^{(2)}(z)\right\|=O\left(\frac{1}{n^{2 \gamma}}+\left|q_{n+1}\right|\right) \quad \text { as } n \rightarrow \infty .
$$

and $\mu_{2}(z)$ with zero. In the proof of Lemma 3.1 , we include terms of the order $O\left(1 / n^{2 \gamma}\right)$ into the remainder and hence make no use of $M_{ \pm 4}(z), X_{ \pm 4}(z), V(z)$ and $T_{n}^{(2)}(z)$. The condition $e^{ \pm i \omega} \neq 1$ is not needed anymore. The system 3.12 becomes

$$
w_{n+1}=\left[\left(\begin{array}{cc}
1 / z & 0 \\
0 & z
\end{array}\right)+R_{n}^{(2)}(z)\right] w_{n} .
$$

In the statement and proof of Theorem 5.1 we can also replace

$$
U, \quad O\left(1 / n^{3 \gamma}+\left|q_{n+1}\right|\right), \quad \mu_{2}(z)
$$


with respectively

$$
\mathbb{C} \backslash\left\{0,1,-1, e^{ \pm \omega},-e^{ \pm \omega}\right\}, \quad O\left(1 / n^{2 \gamma}+\left|q_{n+1}\right|\right), \quad 0 .
$$

Most of the estimates that we use there become trivial. The same modifications should be applied to the proof of Theorem 5.3, together with replacing $(-2 ; 2) \backslash\{ \pm 2 \cos \omega, \pm 2 \cos 2 \omega\}$ by $(-2 ; 2) \backslash\{ \pm 2 \cos \omega\}$.

Acknowledgements. The second author expresses his deep gratitude to Prof. S. N. Naboko for his constant attention to this work and for many fruitful discussions of the subject, to Dr. R. Romanov for his help and interesting discussions and to Prof. V. Bergelson for the detailed explanations of the related subjects. The work was supported by grants RFBR-09-01-00515-a and INTAS-05-1000008-7883.

\section{References}

[1] N. Akhiezer, The Classical Moment Problem and Some Related Questions in Analysis, Oliver \& Boyd, 1965.

[2] H. Behncke, Absolute continuity of hamiltonians with von Neumann Wigner potentials I, Proc. Amer. Math. Soc. 111 (1991), 373-384.

[3] —, Absolute continuity of hamiltonians with von Neumann Wigner potentials II, Manuscripta Math. 71 (1991), 163-181.

[4] Z. Benzaid and D. Lutz, Asymptotic representation of solutions of perturbed systems of linear difference equations, Stud. Appl. Math. 77 (1987), 195-221.

[5] B. Brown, M. Eastham, and D. McCormack, Absolute continuity and spectral concentration for slowly decaying potentials, J. Comput. Appl. Math. 94 (1998), 181197.

[6] D. Damanik and B. Simon, Jost functions and Jost solutions for Jacobi matrices, I. A necessary and sufficient condition for Szegö asymptotics, Invent. Math. 165 (2006), 1-50.

[7] M. Eastham, The Asymptotic Solution of Linear Differential Systems. Applications of the Levinson Theorem, Clarendon Press, Oxford, 1989.

[8] D. Gilbert and D. Pearson, On subordinacy and analysis of the spectrum of onedimensional Schrödinger operators, J. Math. Anal. Appl. 128 (1987), 30-56.

[9] W. Harris and D. Lutz, Asymptotic integration of adiabatic oscillators, ibid. 51 (1975), 76-93.

[10] D. Hinton, M. Klaus, and J. Shaw, Embedded half-bound states for potentials of Wigner-von Neumann type, Proc. London Math. Soc. 3 (1991), 607-646.

[11] J. Janas and M. Moszyński, Spectral properties of Jacobi matrices by asymptotic analysis, J. Approx. Theory 120 (2003), 309-336.

[12] - - -, New discrete Levinson type asymptotics of solutions of linear systems, J. Difference Equations Appl. 12 (2006), 133-163.

[13] J. Janas, S. Naboko, and E. Sheronova, Jacobi matrices arising in the spectral phase transition phenomena: asymptotics of generalized eigenvectors in the "double root" case, Centre for Math. Sciences, Lund Institute of Technology, 2007, mp_arc 07-41.

[14] S. Khan and D. Pearson, Subordinacy and spectral theory for infinite matrices, Helv. Phys. Acta 65 (1992), 505-527. 
[15] M. Klaus, Asymptotic behavior of Jost functions near resonance points for Wignervon Neumann type potentials, J. Math. Phys. 32 (1991), 163-174.

[16] K. Kodaira, The eigenvalue problem for ordinary differential equations of the second order and Heisenberg's theory of S-matrices, Amer. J. Math. 71 (1949), 921-945.

[17] P. Kurasov, Zero-range potentials with internal structures and the inverse scattering problem, Lett. Math. Phys. 25 (1992), 287-297.

[18] -, Scattering matrices with finite phase shift and the inverse scattering problem, Inverse Problems 12 (1996), 295-307.

[19] P. Kurasov and S. Naboko, Wigner-von Neumann perturbations of a periodic potential: spectral singularities in bands, Math. Proc. Cambridge Philos. Soc. 142 (2007), 161-183.

[20] M. Lukic, Orthogonal polynomials with recursion coefficients of generalized bounded variation, 2010, arXiv:1008.3844, mp_arc 10-129.

[21] V. Matveev, Wave operators and positive eigenvalues for a Schrödinger equation with oscillating potential, Theoret. Math. Phys. 15 (1973), 574-583.

[22] M. Moszyński, Slowly oscillating perturbations of periodic Jacobi matrices in $l^{2}(\mathbb{N})$, Studia Math. 192 (2009), 259-279.

[23] S. Naboko, I. Pchelintseva, and L. Silva, Discrete spectrum in a critical coupling case of Jacobi matrices with spectral phase transitions by uniform asymptotic analysis, J. Approx. Theory 161 (2009), 314-336.

[24] S. Naboko and S. Simonov, Spectral analysis of a class of hermitian Jacobi matrices in a critical (double root) hyperbolic case, Proc. Edinburgh Math. Soc. (2) 53 (2010), 239-254.

[25] L. Silva, Uniform Levinson type theorems for discrete linear systems, in: Oper. Theory Adv. Appl. 154, Birkhäuser, 2004, 203-218.

[26] -, Uniform and smooth Benzaid-Lutz type theorems and applications to Jacobi matrices, in: Oper. Theory Adv. Appl. 174, Birkhäuser, 2007, 173-186.

[27] E. Titchmarsh, Eigenfunction Expansions Associated with Second-Order Differential Equations. Part I, Clarendon Press, Oxford, 1946.

Jan Janas

Instytut Matematyczny

Polskiej Akademii Nauk

Św. Tomasza 30

31-027 Kraków, Poland

E-mail: najanas@cyf-kr.edu.pl
Sergey Simonov Department of Mathematical Physics Institute of Physics St. Petersburg University Ul'yanovskaya 1

St. Petergoff, St. Petersburg, 198904, Russia E-mail: sergey_simonov@mail.ru 